\title{
Note de correction : Le déterminisme du sexe chez les cucurbitacées
}

Camille Foucart, Adnane Boualem, Bertrand Lasseur, John Eleblu, Izhak Farhaj, et Abdelhafid Bendahmane*

Inra-CNRS, UMR 1165, Unité de Recherche en Génomique Végétale, 91057 Évry cedex, France

La version en ligne de l'article original peut être trouvée dans: Biologie Aujourd'hui 206(1), 57-62 (2012)

https://doi.org/10.1051/jbio/2012005

Reçu le 29 mai 2017

Une erreur s'est produite dans l'article «Le déterminisme du sexe chez les cucurbitacées 》 publié dans le volume 206 (1), pp. 57-62 (2012). Le nom du cinquième auteur Izhak Farhaj a été mal orthographié (Izhak Fahraj). Le nom correct est Izhak Farhaj. L'éditeur s'excuse pour cette erreur.

\footnotetext{
*Auteur correspondant: bendahm@evry.inra.fr
} 